\title{
Modification of the Discharge of Lateral Geniculate Neurons During Visual Learning
}

\author{
Charles M. Gibbs, David H. Cohen, and James L. Broyles \\ Department of Neurobiology and Behavior, State University of New York at Stony Brook, \\ Stony Brook, New York 11794
}

Visually conditioned heart rate change in the pigeon has been developed as a vertebrate model system for cellular analysis of associative learning. Previous studies have characterized the behavior, largely delineated the neural circuitry mediating the conditioning, and estimated the central processing time for the conditioned response. Most recently, this system has been used to investigate neuronal activity during conditioning along the visual pathways that transmit the conditioned stimulus (CS) information. It was first shown that neither maintained nor CSevoked discharge of retinal ganglion cells changes during conditioning. Subsequently, we found that the thalamic and telencephalic components of the ascending tectofugal pathway show associative modification.

We report here studies of the thalamofugal pathway, the avian homolog of the mammalian geniculocortical system. Singlecell activity was recorded in the thalamic relay of this pathway, the dorsal lateral geniculate equivalent (LGNe). This provided an opportunity to (1) evaluate the generality of the traininginduced modification found along the tectofugal pathway, and to (2) determine if such modification occurs as peripherally as retinorecipient neurons.

The results show that almost all LGNe neurons (97\%) respond phasically to the onset of whole-field illumination. Most $(\mathbf{9 4 \% )}$ also respond to the unconditioned stimulus (US), footshock, some with increased and others with decreased discharge. Of cells receiving convergent input, those responding with decreased discharge to the US showed associative change $(52 \%)$. Neurons that did not respond to both the CS and US, or that responded to the US with increased discharge, did not show associative modification.

These findings suggest that (1) the visual pathways transmitting CS information are not merely input lines, but undergo training-induced modification; (2) such modification can occur as peripherally as the retinorecipient neurons of these pathways; and (3) CS-US convergence is necessary but not sufficient for associative modification, since modifiability is apparently contingent on specific US response properties.

A particularly challenging problem in neurobiology has been to identify sites of information storage during learning, a prerequisite to analyzing cellular mechanisms of training-induced modification. The development of model systems over the past

Received Jan. 10, 1985; revised Apr. 29, 1985; accepted June 11, 1985.

This research was supported by NSF Grant BNS-80-16396 to D.H.C. C.M.G. was partially supported by postdoctoral fellowships from the NSF (PDF-8166055) and NIMH (MH086001). We thank Mr. Alann Solina and Ms. Mary Ellen Fay for their technical assistance and Ms. Adrienne Walter for her secretarial assistance. Portions of this paper were presented previously at the Annual Meeting of the Society for Neuroscience [(1980) Neurosci. Abstr. 6: 424; (1981) 7: 752].

Correspondence should be addressed to Dr. Cohen.

Copyright (C) 1986 Society for Neuroscience $0270-6474 / 86 / 030627-10 \$ 02.00 / 0$ decade or two, particularly of simple invertebrate systems, has generated exciting progress in this regard (e.g., Alkon, 1984; Kandel and Schwartz, 1982). Yet, despite promising recent advances in the development of vertebrate models (e.g., Cohen, 1984; Thompson et al., 1983; Tsukahara, 1982), definitive identification of synaptic fields involved in information storage has been elusive. We describe here a population of neurons that receives convergent $\mathrm{CS}$ and US inputs and may be a primary site of change during conditioning.

The results are from a vertebrate model system we have been investigating for some years - visually conditioned heart rate change in the pigeon (Cohen, 1969, 1974). In developing this system, an effective behavioral paradigm has been extensively characterized (Cohen and Goff, 1978) and progress in describing the relevant circuitry (Cohen, 1980) has been sufficient to allow cellular neurophysiological analysis. The first such studies were of the "cardiac motoneurons," both vagal (Gold and Cohen, 1981a, 1984) and sympathetic (Cabot, 1976; Cohen, 1982). These studies provided an estimate of the central processing time for conditioned responding, a time that is sufficiently short to suggest that the system is quite amenable to neurophysiological analysis (Cohen, 1982).

Motoneuronal discharge change during conditioning is an expected, indeed necessary, result. However, it is difficult to determine the extent to which such changes reflect modification of more central structures and/or plasticity at the motoneurons. Therefore, we directed our attention to the pathways transmitting the CS information (Cohen, 1984). A study of the activity of single retinal ganglion cells during conditioning demonstrated that neither the maintained nor CS-evoked discharge of these neurons changes (Wild and Cohen, 1985). We then explored the tectofugal pathway, one of the prominent ascending visual pathways that transmits effective CS information (Cohen, 1980; Cohen et al., unpublished observations). The data indicated that neurons in both the thalamic and telencephalic components of this pathway show training-induced modification and that the change in CS-evoked discharge parallels the acquisition of the behavioral response (Wall et al., 1985).

Since some tectal neurons are polysensory and respond to somatic as well as visual stimuli, the tectofugal pathway may receive convergent $\mathrm{CS}$ and US inputs. This could establish a substrate for conditioned modification that is unique to that pathway and does not occur in the other visual pathways transmitting CS information.

To evaluate the generality of the changes along the tectofugal pathway, we investigated the thalamofugal system, another ascending visual pathway that transmits effective $\mathrm{CS}$ information (Cohen, 1980; Cohen et al., unpublished observations). This pathway is homologous to the mammalian geniculocortical system (Karten, 1979), and we specifically directed our attention to its thalamic relay, the avian equivalent of the dorsal lateral geniculate nucleus (LGNe). Thus, this study also evaluated 
whether training-induced modification occurs as peripherally as retinorecipient neurons.

\section{Materials and Methods}

\section{Experimental animals}

The experiments involved 83 male and female White Carneaux pigeons (Columba livia), ranging in age from 2 to 6 months and weighing 460$620 \mathrm{gm}$. The birds were experimentally naive at the start of the experiments and were housed individually and maintained on a 12-hr lightdark cycle for at least $7 \mathrm{~d}$ before training.

\section{Experimental preparation}

The feathers around the external auditory meatus and on the dorsal surface of the head were clipped and these areas were liberally infiltrated with a $2 \%$ lidocaine solution. The trachea was intubated with silicon tubing coated with $2 \%$ xylocaine jelly, and neuromuscular blockade was established with $\alpha$-bungarotoxin or $\alpha$-cobratoxin (Gold and Cohen, 1981b). The birds were ventilated to maintain the continuously monitored end-tidal $\mathrm{CO}_{2}$ at $4-5 \%$, and body temperature was maintained with a thermostatically controlled water pad. Following fixation in the stereotaxic apparatus with blunt car bars, a small area of the telencephalon was exposed to permit electrode penetrations of the principal optic nucleus (Karten et al., 1973), the LGNe. The left pupil was dilated by topically applying $d$-tubocurarine chloride $\left(3 \mathrm{mg} / \mathrm{cm}^{3}\right)$ and $0.025 \%$ benzalkonium chloride (Campbell and Smith, 1962). The lower eyelid was retracted, and a zero-power, translucent contact lens was placed over the cornea.

Extreme care was taken with infiltration anesthesia throughout the experiments. It should be noted that successful conditioning demands that the animals be in no distress, since discomfort is invariably reflected in high baseline heart rates and a lack of conditioning.

\section{Recording procedures}

The electrocardiogram was recorded with subdermal electrodes in the base of the wing and back. Lists of the times of occurrence of K-waves were generated by on-line computer.

Electrolytically etched tungsten microelectrodes $(5-15 \mathrm{M} \Omega$ at $500 \mathrm{~Hz})$ were used for recording neuronal activity. Electrode penetrations were initiated after a period of dark adaptation in the experimental chamber, a light-tight, sound-attenuating cubicle. The penetrations were stereotaxically guided and the electrode advanced by hydraulic microdrive. Upon isolating the activity of a single LGNe cell, the conformation of the spikc was noted and then carefully monitored to ensure that the activity of the same cell was recorded throughout the experiment. The signal-to-noise ratio was $5: 1$ or greater, with spike amplitudes of $0.8-$ $5.0 \mathrm{mV}$. Records of neuronal discharge were stored on analog tape, and on-line lists of the times of occurrence were generated by computer.

\section{Histological procedures}

At the completion of each experiment, the recording site was marked by passing a 4-6 $\mu \mathrm{A}$ DC current for $10 \mathrm{sec}$ through the recording electrode. The animal was then killed with chloral hydrate and perfused through an internal carotid artery with saline followed by $10 \%$ formalin. After immersion fixation, brains were removed, blocked stereotaxically in the transverse plane, and cut frozen at $50 \mu \mathrm{m}$. Sections were stained with cresyl violet and the electrolytic lesions charted. Analysis indicated that the recording sites were distributed throughout all the subnuclei of LGNe, being densest in the dorsal and ventral subdivisions of nucleus dorsolateralis anterior thalami, pars lateralis. There were no obvious preferential distributions with respect to either CS or US response classes.

\section{Behavioral procedures}

\section{Stimuli}

The visual stimulus was a $50 \mathrm{ft}$-Lambert $(1 \mathrm{ft}$-Lambert $=3.4263 \mathrm{~cd} /$ $\left.\mathrm{m}^{2}\right), 6 \mathrm{sec}$ monocular presentation of whole-field illumination delivered through a fiber-optic bundle and an electronically controlled shutter (Gold and Cohen, 1981a, 1984). Since there is complete decussation at the pigeon's optic chiasm, all recording was from the LGNe contralateral to the stimulated eye. The US was a footshock consisting of a $500 \mathrm{msec}$ train of biphasic, constant-current pulses at $60 \mathrm{~Hz}$. Current levels were adjusted throughout the experiment to produce heart rate increases of $140-180$ beats/min.

\section{Training protocols}

In the initial study, animals were assigned to either an associative training (conditioning; $n=40$ ) or a nonassociative control (sensitization; $n=24$ ) group. The conditioning group received 10 presentations of light alone, followed by 30 presentations of light immediately followed by the US. The pretraining stimuli were used to characterize the neuronal responses prior to training; such light presentations do not affect subsequent conditioning (Cohen and Macdonald, 1971). The nonassociative group received 10 light presentations as well, but these were followed by 30 lights and 30 shocks that were explicitly unpaired with any two successive stimuli separated by at least $1 \mathrm{~min}$ (Cohen and Goff, 1978). For all animals, the mean intertrial interval for light presentations was $3.7 \mathrm{~min}$, with a range of $2.5-5.0 \mathrm{~min}$.

During conditioning, neuronal responses to the US are confounded with responses to $\mathrm{CS}$ termination. Therefore, to evaluate US responsiveness of LGNe cells, both the conditioning and control groups were given a series of $50 \mathrm{msec}$ footshocks at the completion of training.

The initial study identified a specific population of modifiable $\mathrm{LGNe}$ neurons. A second study was then undertaken to replicate this result, and it included 20 animals in which only neurons with specific response characteristics were sampled (see Results). All animals in this second study were given associative training, and the procedures were identical to the initial study except that a short series of $50-100 \mathrm{msec}$ lights and $50 \mathrm{msec}$ footshocks was given before the pretraining light presentations. This was necessary to classify the neurons for sampling purposes. The additional stimuli would not be expected to affect conditioning (Cohen and Macdonald, 1971), and this was confirmed by statistically comparing the results of the initial and replication studies.

\section{Results}

\section{Experiment I}

\section{Neuronal responses to whole-field illumination}

Responses to the 10 initial light presentations were analyzed for 69 LGNe cells recorded from the 64 birds. Inspection of peristimulus histograms for each neuron indicated heterogeneous and sometimes complex response patterns. However, as in previous studies of the visual pathways (Wall et al., 1985), a straightforward response classification was made possible by restricting analysis to the short-latency, transient responses at light onset. This procedure has independent validity, since the conditioned response (CR) is primarily driven by phasic neuronal activity occurring at CS onset (Cohen, 1982; Wild and Cohen, 1985).

With respect to responses at light onset, 41 cells (59\%) showed a light-evoked increase in discharge (Figs. $1 A$ and 2 ) at latencies of 23-70 msec (mean $\pm \mathrm{SE}=33.2 \pm 1.5 \mathrm{msec}$ ). These "on" bursts had an average of $4.2 \pm 0.5$ spikes and a mean duration of $30.9 \pm 5.7 \mathrm{msec}$ (Fig. 2). Two neurons had no phasic responses, and the remaining 26 cells decreased their discharge at light onset (Fig. $1 B$ ). To confirm these phasic decreases statistically, a window of the poststimulus histogram was defined which bounded the response to a maximum of $500 \mathrm{msec}$. Computing $z$ scores then allowed evaluation of whether the discharge within that window differed significantly from maintained activity (critical value $=1.65$ ). Precise detcrmination of the latencies and durations of these phasic decreases in discharge was difficult because of low levels of maintained activity. However, the decreases were typically apparent within 31-50 msec of light onset (Fig. $1 B$ ) and persisted for 25 to over $500 \mathrm{msec}$.

\section{Neuronal responses to the US}

Of the 67 visually responsive cells, 63 also responded to the US. Thus, $91 \%$ of LGNe neurons received convergent CS and US inputs. Thirty of these (45\%) showed increased discharge (Fig. 3, $A, B$ ) and are denoted US + cells, while 33 (49\%) showed decreased discharge (Fig. 3, $C, D$ ) and are denoted US_ cells. 
A. EVOKED DISCHARGE INCREASE

$$
(n=41)
$$

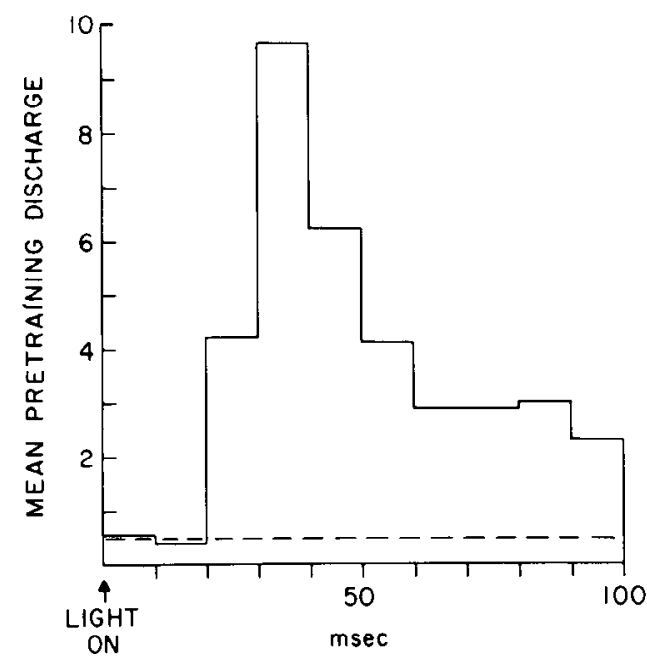

8. EVOKED DISCHARGE DECREASE

$$
(n=26)
$$

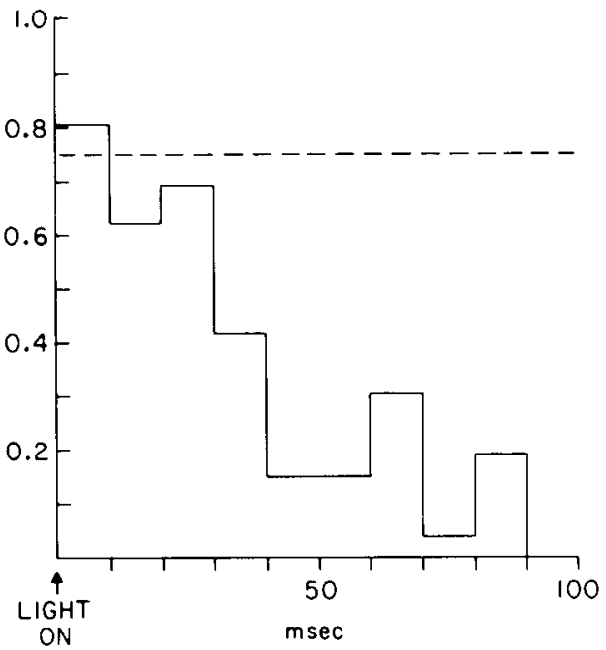

Figure 1. Poststimulus histograms showing light-evoked responses of LGNe neurons during the initial 10 , light-alone presentations (pretraining). $A$, Mean response of neurons with increased discharge. $B$, Mean response of neurons with decreased discharge. Mean maintained activity levels are indicated by the broken horizontal lines. Light onset occurred at $0 \mathrm{msec}$, as indicated by the arrows.
The mean response latency of the US + cells was $105.2 \pm 5.3$ msec, with a range of 70-203 msec. Low levels of maintained activity precluded precise latency estimates of most US_ cells, but all showed clear decreases in discharge within the latency range of the US, cells. Thus, the latency distributions of the two US-rcsponsc classes are largely overlapping. Finally, there was no statistically significant relationship between the directions of the light-evoked and US-evoked responses $\left[\chi^{2}(2)=1.38, \mathrm{~ns}\right]$.

\section{Effects of training on phasic, light-evoked responses}

To assess the effects of associative and nonassociative training on the light-evoked activity of LGNe cells with increased discharge, the number of spikes in the phasic response of each cell was averaged for the 10 pretraining trials and for each 10 -trial training block. The 10-trial means during training were then expressed as percentage changes from the mean response during pretraining. For cells with decreased discharge, the number of spikes in the phasic response window was averaged over the corresponding pretraining and training blocks and normalized with respect to mean maintained activity levels. These normalized 10-trial means during training were then expressed as percentage changes from those in pretraining.

The distribution of the 69 LGNe neurons with respect to CS and US response classes and behavioral treatment is shown in Table 1. The two neurons with no phasic response at light onset were both in the conditioning group, and neither showed any change over training.

Of the 41 neurons with excitatory responses to the light, 25 were in the conditioning group (Table 1), and nine showed reliable enhancement of their CS-evoked discharge over training (e.g., Fig. 9). In contrast, five of the 16 cells in the control group showed no change in light-evoked discharge, and the discharge of the remaining 11 was attenuated (e.g., Fig. 9). This differential effect of associative and nonassociative training (Fig. $4 A$ ) was confirmed by an analysis of variance that indicated a significant main effect due to Groups $(F(1,39)=8.88, p<0.005)$ and a significant Groups $\times$ Blocks interaction $(F(2,78)=3.71, p<$ 0.05 ). It should be noted that the two groups did not differ statistically during pretraining, either in maintained activity or light-evoked responses (i.e., latency, number of discharges, total burst duration). Moreover, statistical analysis indicated that maintained activity did not change in either group over training.

Of the 26 neurons that responded at light onset with decreased discharge, 17 were in the conditioning group and nine in the control group (Table 1). The responses of neurons in the conditioning group essentially remained at pretraining levels throughout conditioning (Fig. $4 B$ ). In contrast, responses of cells in the nonassociative group attenuated rapidly (Fig. $3 B$ ). This differential effect was confirmed by analysis of variance $(F(1,24)=12.61, p<0.005)$, and a block-by-block analysis in-

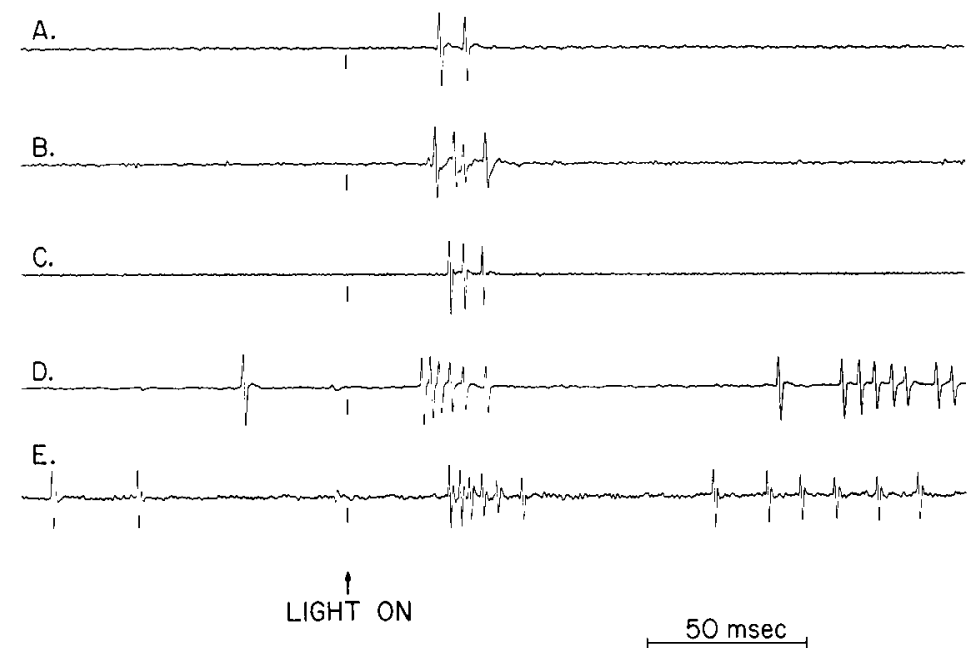

Figure 2. Examples of excitatory responses at light onset. Shown are the responses of five different LGNe cells to a single light presentation during pretraining. Note the well-defined "on" bursts. 


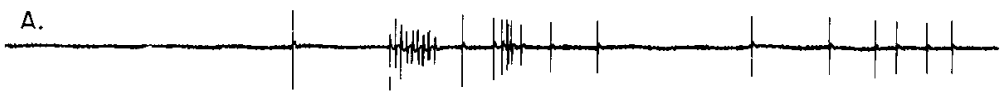

Figure 3. Examples of responses to the US, footshock. Shown are the responses of four different LGNe cells to a single $50 \mathrm{msec}$ footshock delivered at the end of training. $A$ and $B$, Representative responses of cells with increased discharge (US $)_{+}$). $C$ and $D$, Representative responses of cells with decreased discharge (US_). Note that the spikes during the $50 \mathrm{msec}$ period following SHOCK $O N$ are shock artifact.
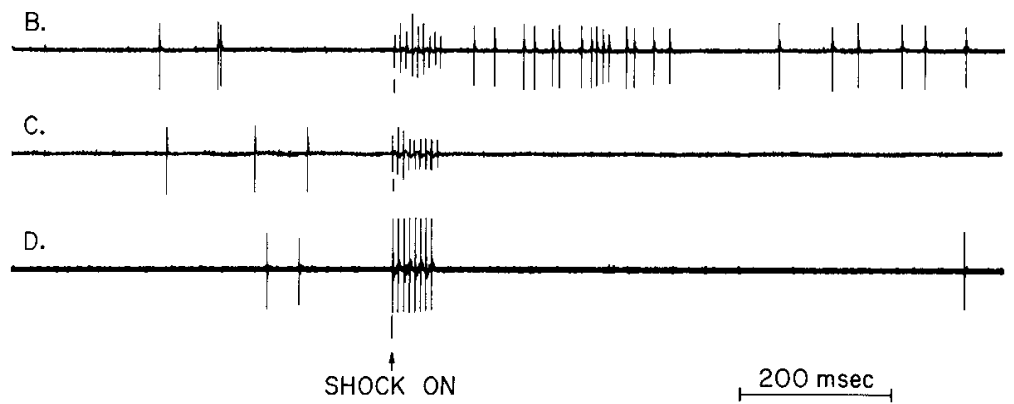

dicated that the group differences emerged within 10 trials $(t(24)=3.67, p<0.001)$. These effects were confounded neither by group differences in pretraining nor by differential changes in maintained activity.

Thus, LGNe neurons unresponsive to whole-field illumination do not show training-induced modification. Neurons with a phasic decrease in discharge at light onset maintain that response over associative training, while it attenuates with nonassociative training. Approximately one-third of the neurons with phasic increases in discharge at light onset show enhancement of this response over associative training, while the response either does not change or attenuates during nonassociative training.

\section{Role of US responsiveness in training effects}

Only four of the LGNe neurons did not respond to the US (Table 1); these are designated $\mathrm{US}_{0}$ cells. The remaining neurons divided approximately equally between $U_{+}$and US_cells. Thus, there are three classes of LGNe neurons with respect to US responsiveness, and more detailed analysis taking this into ac-

A. INCREASED DISCHARGE

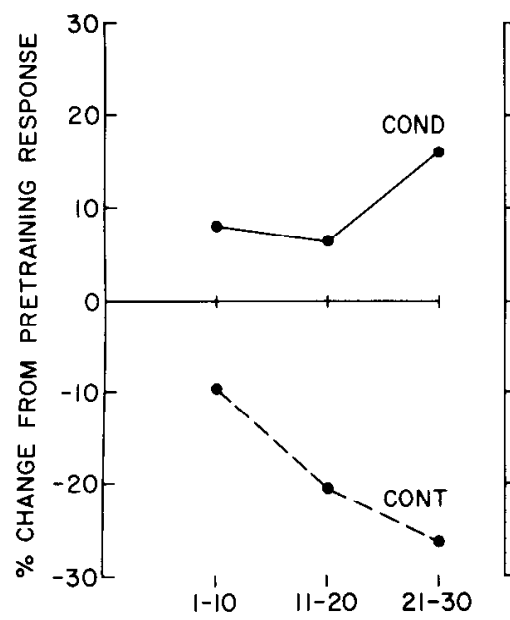

TRIALS
B. DECREASED DISCHARGE

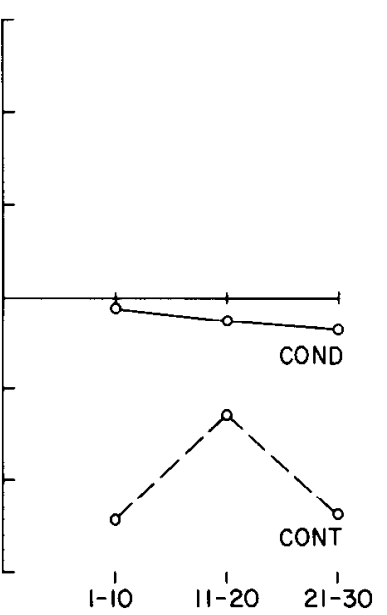

Figure 4. Mean changes in light-evoked activity of LGNe neurons during conditioning $(C O N D)$ and nonassociative control $(C O N T)$ training. $A$, Summary of results for cells responding with increased discharge at light onset $\left(n=41\right.$; Table $\left.1, C_{+}\right) . B$, Summary of the cells responding with decreased discharge $\left(n=26\right.$; Table $\left.1, \mathrm{CS}_{-}\right)$. The response levels for each 10-trial block of training are expressed as a percentage change from the 10-trial pretraining block. Positive percentages reflect enhancement of the pretraining response, be it increased or decreased discharge, while negative percentages reflect attenuation of the initial response. count showed that US responsiveness is critical in traininginduced modification.

The four $\mathrm{US}_{0}$ neurons were in the conditioning group (Table 1) and had excitatory responses to the CS. None showed response enhancement (Fig. 5). Of the remaining 21 cells in the conditioning group that showed CS-evoked increases in discharge, 12 were US + neurons and nine US_(Table 1). Analyzing these two US-responsive groups separately demonstrated a clear differential effect (Fig. 5). The nine neurons showing response enhancement were all in the US group. In contrast, the 12 cells in the US, group showed either no change or response attenuation over training (Fig. 5). Analysis of variance of the 25 cells with CS-evoked increase in discharge in the conditioning group, separating for US class, confirmed this differential effect $(F(2,22)=19.53, p<0.001)$.

A similar analysis was not feasible for the 17 neurons in the conditioning group with decreased discharge to the CS (Table

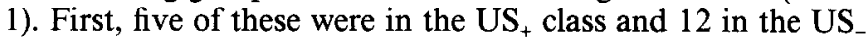
class; only five cells was insufficient for reliable statistical analysis. Second, most cells responding with decreased discharge showed total cessation of activity at light onset before training, precluding any demonstration with extracellular recording of response enhancement. This "floor" thus restricted the range of any group difference that might have obtained between US $_{+}$and US - neurons. Consequently, we were unable to evaluate statistically whether the above differential effect, as a function of USresponse class, occurs for units whose CS-evoked response is a decrease in discharge.

US-response class was not a relevant dimension for animals in the nonassociative control group.

\section{Conditioned heart rate change}

The above results indicate that LGNe neurons with CS-evoked excitatory responses show the clearest associative enhancement. The 24 animals in which such cells were recorded also had appropriate behavioral responses (Fig. 6). The conditioned heart

Table 1. Distribution of cells with respect to CS and US response class and behavioral treatment

\begin{tabular}{|c|c|c|c|c|c|c|c|}
\hline & \multicolumn{3}{|c|}{ Conditioning } & \multicolumn{3}{|c|}{ Control } & \\
\hline & $\overline{\mathrm{CS}_{+}}$ & $\mathrm{CS}_{-}$ & $\mathrm{CS}_{0}$ & $\overline{\mathrm{CS}_{+}}$ & $\mathrm{CS}_{-}$ & $\overline{\mathrm{CS}_{0}}$ & \\
\hline $\mathrm{US}_{+}$ & 12 & 5 & 1 & 9 & 5 & 0 & 32 \\
\hline US & 9 & 12 & 1 & 7 & 4 & 0 & 33 \\
\hline $\mathrm{US}_{0}$ & 4 & 0 & 0 & 0 & 0 & 0 & 4 \\
\hline Total & 25 & 17 & 2 & 16 & 9 & 0 & 69 \\
\hline
\end{tabular}

Distribution of the 69 neurons of Experiment I with respect to phasic responsiveness to the CS and ISS (+, increased discharge; - , decreased discharge; 0 , unresponsive). The neurons are also divided with respect to behavioral treatment (Conditioning $=$ associative training; Control $=$ nonassociative training). 


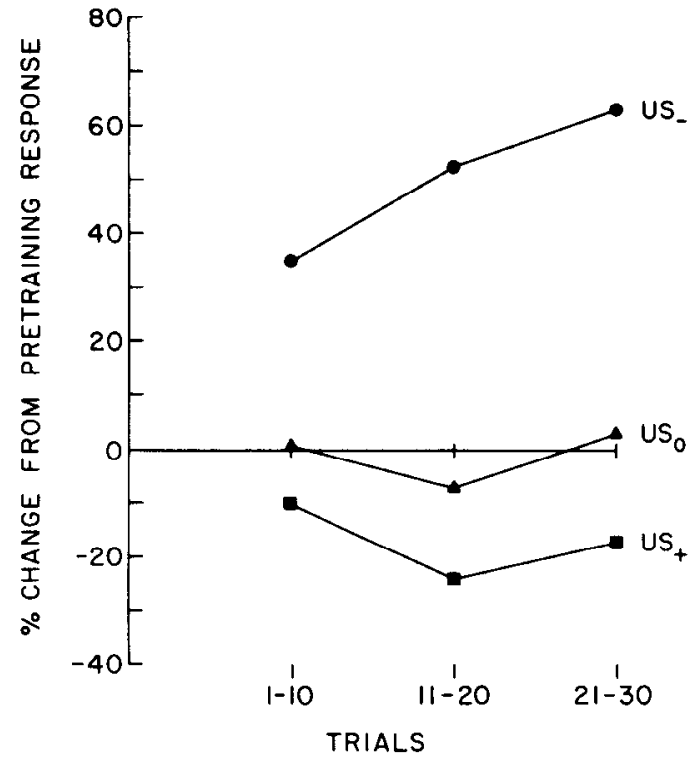

Figure 5. Training-induced modification as a function of response to the US. Shown are the data for cells with CS-evoked increases in discharge $(n=25)$. These cells have been separated into three groups with respect to US responsiveness (see Table 1): unresponsive $\left(\mathrm{US}_{0} ; n=4\right.$ ), increased discharge (US $; n=12$ ), and decreased discharge (US $; n=$ 9). The response levels for the 10-trial training blocks are expressed as a percentage change from the 10-trial pretraining block. Positive percentages reflect enhancement of the pretraining responses, while negative percentages reflect attenuation of the initial response. Note that associative modification is restricted to the US group of LGNe neurons.

rate change of this group is shown in comparison to the responses of the 14 control animals (Fig. 6). (Data for one control animal were unavailable because of equipment malfunction.)

These associative and nonassociative groups did not differ with respect to heart rate responses during pretraining. However, over successive training blocks, the conditioning group showed increased heart rate change, while the control group progressively habituated. Analysis of variance confirmed this differential effect, yielding a reliable effect of Groups $(F(1,36)=$ $6.41, p<0.025)$ and a significant Groups $\times$ Blocks interaction $(F(2,72)=7.07, p<0.005)$. Since the groups did not differ with respect to baseline heart rate, the data establish that associative learning occurred.

\section{Experiment II}

Experiment I showed that neurons unresponsive to the US or unresponsive phasically to the CS do not show associative modification, which suggests the necessity for CS-US convergence. However, Experiment I also suggested that such convergence is not sufficient, since conditioned enhancement of the CS-evoked response occurred only in US_ cells. Since that subsample consisted of only nine cells, a replication experiment was undertaken. Twenty additional neurons were investigated during conditioning, all with phasic excitatory responses to the CS. Ten were in the US class, and, of the remaining 10, six were $\mathrm{US}_{+}$ cells and four $\mathrm{US}_{0}$.

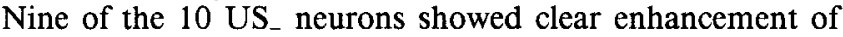
CS-evoked discharge over training, and $t$ tests for each of the 10 -trial training blocks confirmed this effect (all $t$ values $\simeq 3.8$, $p<0.005)$. In contrast, CS-evoked responses of the $\mathrm{US}_{+}$and $\mathrm{US}_{0}$ cells either attenuated or did not change over training. Analyses of variance confirmed that the responses of US cells differed significantly from both $\mathrm{US}_{+}(F(1,14)=14.35, p<0.01)$ and $\operatorname{US}_{0}(F(1,12)=14.35, p<0.01)$ cells. There were no group differences or changes in maintained activity. Thus, the results were consistent with those of Experiment $\mathrm{I}$.

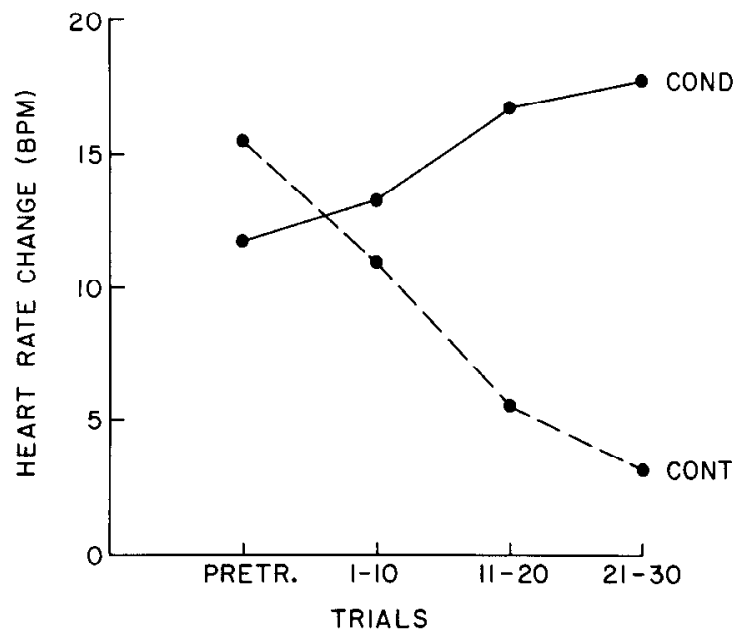

Figure 6. Acquisition of conditioned heart rate change in the group of animals in which excitatory, light-evoked neuronal responses were evaluated. The solid line shows the performance of the conditioning (COND) group, the broken line the performance of the nonassociative control (CONT) group. Each point represents a group mean and shows the heart rate change in beats/min $(B P M)$ between the $6 \mathrm{sec}$ light and the immediately preceding $6 \mathrm{sec}$ control periods. PRETR., The heart rate response during the initial 10-trial block, where both groups received light-alone presentations. Subsequent points represent 10-trial blocks of training.

\section{Pooled sample}

Detailed comparison of the results of Experiments I and II indicated no statistical differences. For example, neurons with CSevoked increases in discharge had mean maintained activity levels of $5.0 \pm 0.4 \mathrm{~Hz}$ in Experiment $\mathrm{I}$ and $4.6 \pm 0.5 \mathrm{~Hz}$ in



Figure 7. The solid line shows modification over conditioning (COND) for the pooled sample of 19 LGNe cells with increased discharge to the CS and decreased discharge to the US (US _ cells). This is compared with a nonassociative control group (CONT) of 16 cells (broken line) with increased discharge to the light. Responses are shown as percentage changes from pretraining, and each point represents a group mean for the trial block indicated along the abscissa. The error bars represent \pm 1 SEM. Positive percentages reflect enhancement of the pretraining response, while negative percentages reflect attenuation. Note that the differential effects of associative and nonassociative training are evident within the first 10 training trials. 


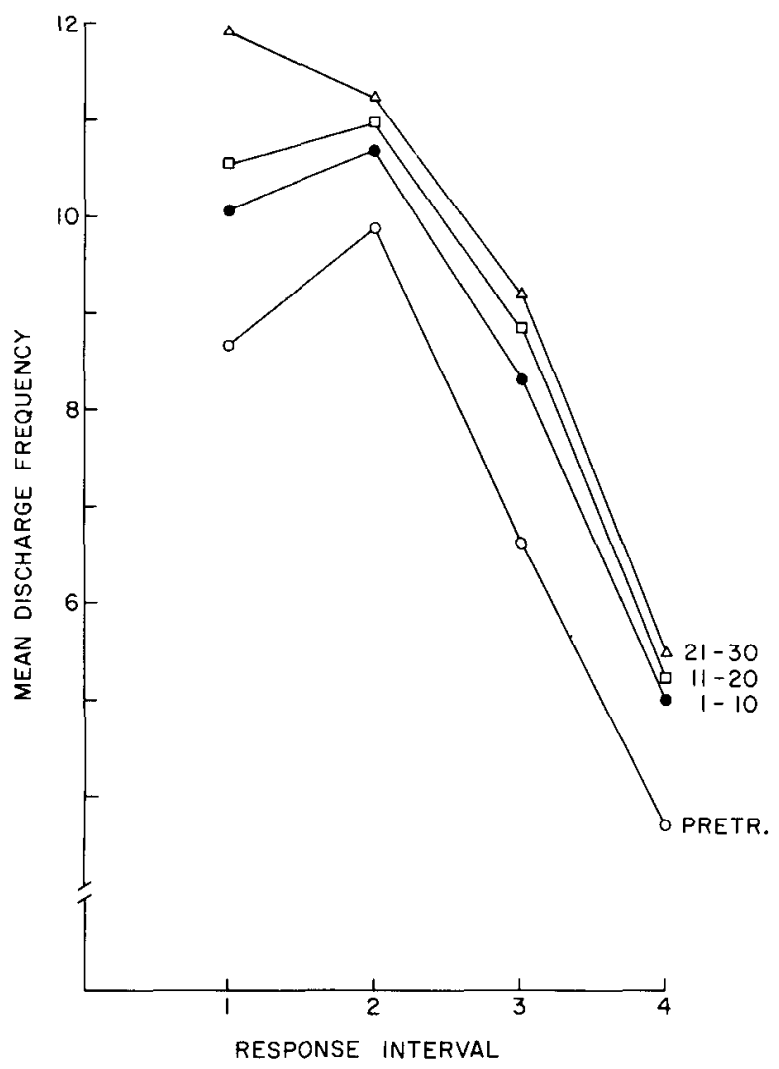

Figure 8. Density of discharge of the 19 modifiable US cells (shown in Figure 7) during successive (equal) periods of their "on" burst. Shown are group means for the 10-trial pretraining (PRETR.) period and for each 10 -trial block of training. Note the prominence of the traininginduced enhancement in the earliest response intervals.

Experiment II. The mean response latency of these cells was $33.2 \pm 1.5 \mathrm{msec}$ in Experiment I and $31.6 \pm 1.3 \mathrm{msec}$ in Experiment II, the two means not differing significantly. Given this, the data from the two experiments were pooled. This gave a conditioning sample of 19 neurons with increased discharge to the CS and decreased discharge to the US. The 16 nonassociative cells of Experiment I with CS-evoked increases in discharge were used as a control group. These neurons distributed over the different US-response classes, but that is not relevant in nonassociative training.

\section{Training effects on phasic light-evoked discharge}

The larger sizes of the groups in the pooled sample allowed more detailed analysis of training effects. In particular, they permitted statistical evaluation of the early training trials, during which much of the modification occurred. Figure 7 summarizes the results, showing each training block with the initial 10 trials divided into two-trial blocks. It is evident that the associative and nonassociative groups did not differ in the first two-trial block. However, they rapidly diverged and differed significantly from trials 5-6 onward (all $p$ values $<0.01$ ). The enhancement of most conditioning cells $(74 \%)$ was asymptotic by the end of the first 10 trials. At that point, responses were $40 \%$ above pretraining levels, and an additional increase of only $10 \%$ occurred over the remainder of training. Analogously, nonassociative cells showed response attentuation of approximately $20 \%$ by the end of the first 10 trials, and this increased to only $25 \%$ by the end of training. Thus, both the associative enhancement and the nonassociative attenuation occurred primarily within the first 10 training trials.

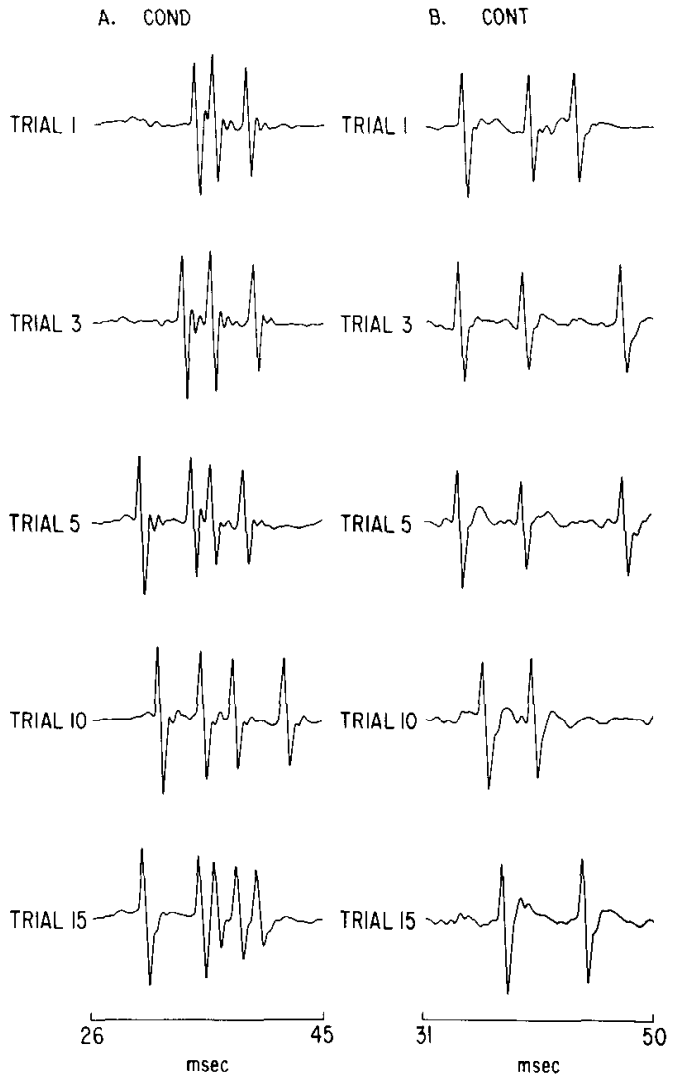

Figure 9. Examples of the behavior of two different LGNe neurons during conditioning (COND) and nonassociative training (CONT). The training trial from which each record was sampled is indicated to the left of that record. The bar below each panel indicates the time period after CS onset for the records shown.

The above results are for the number of discharges in the "on" burst. Similar changes occurred in the duration of the burst. In the conditioning group, the burst duration was $25 \mathrm{msec}$ during pretraining, and this increased to $30 \mathrm{msec}$ over conditioning. In the control group, the initial burst duration was 20 $\mathrm{msec}$, decreasing to $16 \mathrm{msec}$ as the response attenuated. Analysis of variance indicated that this group difference was reliable $(F(1,33)=12.13, p<0.005)$. In addition, response latency decreased by $2.5 \mathrm{msec}$ in the associative group, but increased by $1.3 \mathrm{msec}$ with nonassociative training. Although small, this difference was nonetheless statistically significant $(F(1,33)=8.89$, $p<0.01$ ).

Given these changes in the "on" burst, we analyzed it further to determine if conditioned enhancement occurs preferentially during any particular segment of that burst. For each cell in the conditioning group, a composite response was determined by summing all discharges over the 10 pretraining trials. Each composite was then subdivided into four equal intervals, each successive interval thus representing a later segment of the response. Over the 19 cells, the interval length ranged from 4 to $20 \mathrm{msec}$, with a mean of $10 \mathrm{msec}$. The intervals determined from the pretraining period for each cell were then applied to each training block for that cell, and the discharge frequencies were calculated for the various response segments in both pretraining and training blocks. Averaging these data for the 19 cells then gave the results shown in Figure 8. The discharge density in the response during pretraining is greatest in the first two response segments. During the initial training block, when most modification occurs, the increased discharge distributes rather equally over all four response segments, being only slightly greater in 
the first segment. However, discharge increases later in training seem to occur preferentially in the initial response segment.

Thus, associative response enhancement of $\mathrm{LGNe}$ neurons has the following properties: (1) The number of action potentials in the "on" discharge increases; (2) this increase is evident through the entire "on" burst early in training, but occurs preferentially early in the burst in later training; (3) the latency of the response shortens slightly; (4) the duration of the burst increases; and (5) these changes occur predominantly within the first 10 training trials. This last point is particularly important, since it indicates that neuronal modification in LGNe precedes or occurs in parallel with acquisition of the behavioral CR. Moreover, these changes in both associative and nonassociative groups are clearly evident on an individual cell basis (Fig. 9).

\section{Multiple cells in single experiments}

In Experiments I and II there were six animals in which two discriminable cells were recorded simultaneously, five of the six animals being in a conditioning group. In two of the five, both cells responded similarly to the CS and US, and over training, the pairs were affected similarly. In two other experiments, both cells of each pair were in the US class, one showing increased discharge to the CS and the other decreased discharge. The cell of each pair with increased discharge to the CS showed conditioned enhancement, while the cell of each pair with decreased discharge maintained that response over training. In the remaining experiment, both cells showed increased discharge to

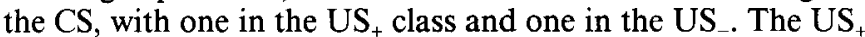
cell showed response attenuation over training, while the US cell showed enhancement. These results indicate that the various response classes can be found in neighboring LGNe neurons. They also demonstrate that discharge changes over conditioning of the different classes can be shown in a single animal.

\section{Training effects on responses during sustained illumination}

To this point, analyses have dealt with phasic responses to light onset, since that response component is most obviously affected by training. The pooled sample provided an opportunity to analyze responses during sustained illumination and to evaluate statistically if they were affected by training.

Of the 61 cells in Experiments I and II with phasic excitatory responses to the light, 24 (39\%) showed no response during sustained illumination (501-6000 msec after light onset). The remaining 37 responded in some manner, most (76\%) with late excitatory responses (see Fig. 2, D,E). Similarly, $65 \%$ of the cells with phasic decreases in discharge showed some response during sustained illumination, generally a late decrease in discharge.

The effects of training on such late responses are illustrated in Figure 10. It is clear that they are not differentially affected by associative and nonassociative training, attenuating in both groups. The results for cells with increased discharge are combined with those of cells with decreased discharge in this illustration, since responses to sustained illumination attenuated regardless of their direction. Thus, the conditioned enhancement of LGNe neurons in the US - class is restricted to the short latency phasic response to the CS.

\section{Discussion}

\section{Plasticity of conditioned stimulus pathways}

How general is the phenomenon?

Our results indicate that a population of LGNe neurons undergoes modification of CS-evoked discharge as a function of associative training. This is most evident in cells with increased discharge to the CS and decreased discharge to the US. Such neurons show robust enhancement of their CS-evoked responses. Cells with decreased discharge at CS onset largely cease

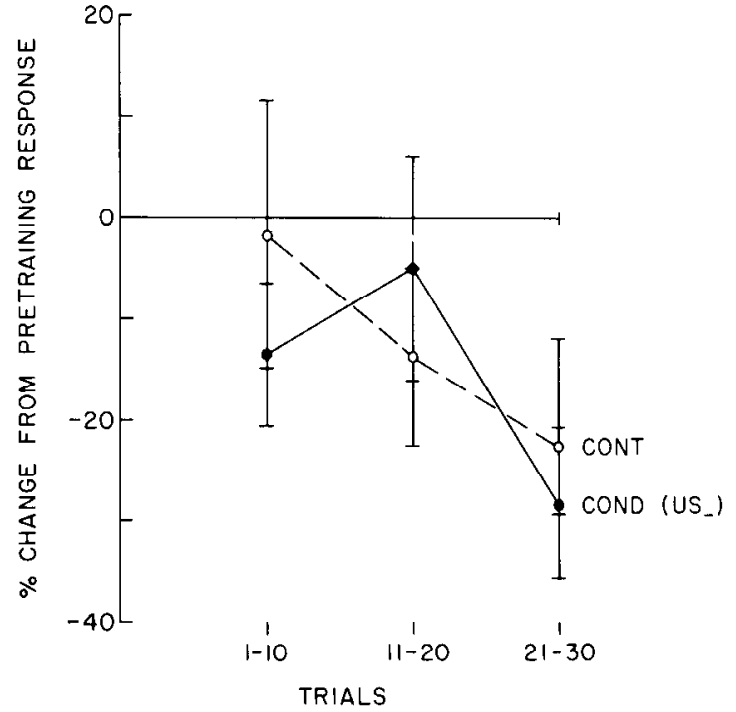

Figure 10. Effect of associative (COND) and nonassociative (CONT) training on the discharge of LGNe during sustained illumination, that is, after the short latency "on" burst. The solid line shows the discharge during conditioning of cells with increased discharge to the CS and decreased discharge to the US (US_ cells), the group showing enhancement of their phasic responses to the CS. The broken line represents the discharge during nonassociative training of a group of cells with increased discharge to the light. Each point represents a group mean percentage change from pretraining during the period 501-6000 $\mathrm{msec}$ after light onset. The error bars indicate \pm 1 SEM. Note that response attenuation occurs irrespective of the training paradigm.

discharging, and thus further reduction in discharge (i.e., response enhancement) could not be demonstrated. However, if such cells also have decreased discharge in response to the US, they maintain their initial light-evoked response over conditioning. In contrast, during nonassociative training, this response attenuates (becomes habituated), suggesting an associative effect. A similar phenomenon was found in an earlier study of the tectofugal pathway (Wall et al., 1985), where it was suggested that associative change for such neurons is reflected as a lack of habituation.

Nonassociative training never resulted in response enhancement, gencrally producing attenuation. Similar behavior was found in LGNe neurons that were either unresponsive to or excited by the US. Consequently, we suggest that all LGNe cells with decreased discharge to the US (US _ cells) are capable of associative modification. These findings extend the generality of earlier results for the tectofugal pathway (Wall et al., 1985) and establish that at least two of the three identified CS pathways (Cohen, 1980) show associative modification.

Plasticity of sensory pathways is well-established in both developing (e.g., Sherman and Spear, 1982) and adult (Killackey and Belford, 1980; Merzenich et al., 1984) nervous systems. With respect to sensory plasticity during learning, invertebrate systems, such as Aplysia (Kandel and Schwartz, 1982) and Hermissenda (Alkon, 1984), show such plasticity as peripherally as primary sensory neurons. However, results for vertebrate models are less consistent. Most studies have used an auditory CS, and in a recent review of that literature, Thompson et al. (1984) cite positive and negative results for all levels of the auditory pathways in one study or another. For example, Kettner and Thompson (1982) report that neurons along the CS pathway show no modification during nictitating membrane conditioning in the rabbit. Similarly, it is reported that the lemniscal portion of the medial geniculate nucleus does not show modification during pupillary conditioning in the cat (Ryugo and Weinberger, 1978), avoidance conditioning in the rabbit (Gabriel et al., 1976), or 
appetitive conditioning in the rat (Birt and Olds, 1981). In contrast, Disterhoft and Stuart (1977) found modification of cells in various auditory structures during appetitive conditioning in the rat. Oleson et al. (1975) also described changes during conditioned pupillary change in the cat. However, neuronal changes in auditory cortex preceded those in the cochlear nucleus, and modification of medial geniculate cells was found only in the nonlemniscal portion of that nucleus. Whether sensory pathways are modified during learning is perhaps a function of the particular behavioral model and/or the nature of the training situation.

\section{Does modification occur at the retinorecipient neurons?}

Our results also suggest that change can occur as peripherally as the retinorecipient neurons. There are, however, two questions in this regard. First, are the modifiable LGNe neurons in fact retinorecipient? Second, does the modification occur in LGNe neurons or in their inputs?

With respect to the first question, the literature on mammals suggests that all neurons of the dorsal lateral geniculate are retinorecipient (Dubin and Cleland, 1977). While there has been no detailed comparison of avian and mammalian lateral geniculate nuclei, it would seem probable that most LGNe neurons are retinorecipient. Further, since about $50 \%$ of LGNe cells were modifiable, at least some, in all likelihood, were retinorecipient.

With regard to the second question, we feel that the discharge changes of LGNe neurons are not secondary to modified inputs. Modification secondary to changes in tonic inputs seems unlikely, since the maintained activity of LGNe cells does not change over training and the phasic and tonic components of the CS-evoked response are differentially affected by conditioning. Stimulus-locked inputs that might modify and phasically affect LGNe neurons would have to do so at very short latency, since only the phasic response to the CS shows associative enhancement, and this is evident in its shortest latency components. A possible source of such input is the retina, but neither the maintained nor CS-evoked discharge of retinal ganglion cells changes over training (Wild and Cohen, 1985). Other possible sources do not respond to the CS at short enough latency. For example, neurons in locus coeruleus often respond to wholefield illumination. However, the mean response latency is 59 msec, and the modified discharge of LGNe neurons is evident within 30-60 msec after CS onset. Similarly, a CS-evoked influence via the projection of the visual Wulst back on the LGNe would be of too long a latency (Britto, 1978).

We cannot exclude the possibility of modification in local, perigeniculate neurons that (1) receive a CS input from LGNe collaterals, (2) receive a US input from the locus coeruleus, and (3) project back on LGNe. While such LGN "interneurons" receiving a brain stem input are reported in the cat (Ahlsen et al., 1984), homologous local circuit neurons for the avian I.GNe have not been described. Moreover, anatomical and electrophysiological evidence (Cohen et al., 1982; Gibbs et al., 1983) indicates a direct effect of locus coeruleus on LGNe neurons, and it thus seems most parsimonious to suggest that LGNe cells are the primary site of modifiability.

\section{Properties of LGNe response modification}

Light-evoked responses of LGNe neurons could be readily segregated into a phasic component at light onset and a tonic component during sustained illumination. This has also been observed in the retinal ganglion cells (Wild and Cohen, 1985), tectofugal pathway (Wall et al., 1985), and cardiac motoneurons (Cohen, 1982; Gold and Cohen, 1981a, 1984). Regardless of their response characteristics to the CS and US and irrespective of training paradigm, tonic responses of LGNc ncurons are similar - they attenuate or remain invariant over training.
We interpret this as habituation, despite its occurrence in both associative and nonassociative training.

In contrast, phasic responses can be differentially affected by associative and nonassociative training. LGNe cells in the $\mathrm{US}_{0}$ and $\mathrm{US}_{+}$classes show attentuation (habituation) of phasic responses. However, US cells show phasic response enhancement with associative training and attenuation with nonassociative training. Thus, associative modification is restricted to the phasic response component, a phenomenon previously observed in the tectofugal system (Wall et al., 1985). This is consistent with the hypothesis (Cohen, 1982, 1984; Wild and Cohen, 1985) that the short-duration "on" burst evoked in the retinal ganglion cells by the CS traverses the participating pathways to be expressed as a phasic response at the motoneurons.

Analysis of phasic responses of modifiable neurons indicated a slight decrease in latency, an increase in the number of discharges in the burst, and a lengthening of the response duration over conditioning. This enhancement developed rapidly, much of it occurring within the first 10 training trials. This directly parallels the acquisition of the behavioral response (Cohen and Goff, 1978), suggesting that neuronal changes in LGNe may contribute to acquisition of the heart rate CR. During this early training period, enhancement of the phasic response was evident throughout its duration. However, the small additional enhancement seen in later training was primarily confined to the initial segment of the responsc. Consequently, throughout training the discharge changes occur in the shortest latency components of the phasic response.

\section{Is CS-US convergence necessary and sufficient?}

\section{Necessity}

A common assumption in neuronal models of classical conditioning is that a modifiable neuron must receive convergent CS and US inputs in an appropriate temporal order. In the present study, the two LGNe neurons unresponsive to the CS did not become responsive over training, suggesting that an initial CS input is necessary. However, if the cell responded to the CS, the characteristics of that response were irrelevant with respect to modifiability. LGNe cells unresponsive to the US were also unmodifiable, suggesting that US input is also necessary. Thus, the data support the hypothesis that CS-US convergence is necessary for associative modification.

\section{Sufficiency}

However, not all cells receiving convergent CS and US inputs showed associative modification. Only those neurons with decreased discharge to the US appeared modifiable. Thus, while CS-US convergence is necessary, it is not sufficient. Apparently, only neurons with specific US response properties are capable of associative modification, suggesting that the US response characteristics are more critical than the CS response properties. Of interest in this regard is that the input mediating the US response probably arises from locus coeruleus, while $\mathrm{US}_{+}$responses can be activated via various projections to the LGNe (Cohen et al., 1982; Gibbs et al., 1983).

\section{Locus of the effect: pre- or postsynaptic?}

An issue deserving discussion is whether the US-evoked decrease in LGNe discharge is due to direct postsynaptic inhibition. As to other possibilities, one is that the effective US input is on terminals of retinal ganglion cells and that decreased LGNe discharge reflects disfacilitation. While ultrastructural data on LGNe are unavailable, mammalian studies indicate no conventional synapses on optic tract terminals in LGN (Guillery, 1971). Another possibility is that the source of the effective US input provides a maintained excitatory influence on LGNe that 
is inhibited by the US, again decreasing LGNe discharge by disfacilitation. However, ongoing studies of locus coeruleus indicate that (1) $81 \%$ of the cells are excited by the US; (2) electrical stimulation of locus coeruleus can decrease the discharge of LGNe neurons; and (3) interruption of the coeruleogeniculate projection does not affect the maintained activity of LGNe neurons (Cohen et al., 1982). Finally, it is possible that the terminals of the projection from locus coeruleus do not form synaptic complexes with postsynaptic elements (Beaudet and Descarries, 1978). Although information in this regard is unavailable for the LGN of either birds or mammals, in a recent review Foote et al. (1983) concluded that locus coeruleus generally forms specialized synaptic contacts with target neurons. Moreover, where evaluated, inhibitory effects of locus coeruleus are reported to result from direct postsynaptic inhibition (e.g., Hoffer et al., 1973). Thus, we favor the interpretation that the US input to LGNe is on retinorecipient neurons and produces direct postsynaptic inhibition.

\section{Some implications of LGNe modifiability}

The finding of associative modification as pcripheral as retinorecipient neurons raises various issues. Since such neuronal changes occur prior to or in parallel with the behavioral $C R$, they can contribute to its development. However, it seems unlikely they are sufficient to mediate that change entirely. In fact, they may not cven be necessary for acquisition of asymptotic performance. This issue deserves further investigation, and it could be approached by selectively eliminating the effective US input to the CS pathways to render them unmodifiable but still capable of transmitting CS information.

Another issue concerns the features of visual information processing that might be modified. It seems unlikely that the pattern recognition properties of LGNe neurons are modified. Perhaps conditioning merely increases the gain of LGNe responses to luminance. Foote et al. (1983) hypothesize that locus coeruleus input to sensory pathways enhances the signal-tonoise ratio of relevant sensory inputs. If this supposition is correct, then one could view modification of the CS pathways as increasing the effectiveness of the CS and thereby facilitating associative modification of more central structures. We have experiments in progress aimed at describing the receptive fields of LGNe neurons and at determining what features of these fields might be affected during conditioning.

Finally, our neurophysiological analysis of this system indicates that phasic responses to the CS preferentially undergo associative enhancement. This implies that some sort of heterosynaptic facilitation occurs at one or more sites along the relevant pathways during training (Cohen, 1984). The present data support an earlier suggestion (Cohen, 1984) that such facilitation is likely to be found at many, if not all, central sites along the pathway. That is, the information storage mediating acquisition of the $C R$ is localized in that it is restricted to the relevant neuronal circuitry, but, within that circuitry, changes may be distributed throughout.

\section{References}

Ahlsen, G., S. Lindstrom, and F.-S. So (1984) Inhibition from the brainstem of inhibitory interneurones of the cat's dorsal lateral geniculate nucleus. J. Physiol. (Lond.) 347: 593-609.

Alkon, D. L. (1984) Calcium-mediated reduction of ionic currents: A biophysical memory trace. Science 226: 1037-1045.

Beaudet, A., and L. Descarries (1978) The monamine innervation of rat cerebral cortex: Synaptic and non-synaptic axon terminals. Neuroscience 3: 851-860.

Birt, D., and M. E. Olds (1981) Associative response changes in lateral midbrain tegmentum and medial geniculate during differential appetitive conditioning. J. Neurophysiol. 46: 1039-1055.

Britto, L. R. G. (1978) Hyperstriatal projections to primary visual relays in pigeons: Electrophysiological studies. Brain Res. 153: 382386.

Cabot, J. B. (1976) Anatomical and electrophysiological analysis of the sympathomotor pathway mediating heart rate conditioning in the pigeon. $\mathrm{Ph}$.D. thesis, University of Virginia, Charlottesville.

Campbell, H. S., and J. L. Smith (1962) The pharmacology of the pigeon pupil. Arch. Ophthalmol. 67: 141-144.

Cohen, D. H. (1969) Development of a vertebrate experimental model for cellular neurophysiologic studies of learning. Cond. Ref. 4: 6180.

Cohen, D. H. (1974) The neural pathways and informational flow mediating a conditioned autonomic response. In Limbic and Autonomic Nervous Systems Research, L. V. DiCara, ed., Plenum, New York.

Cohen, D. H. (1980) The functional ncuroanatomy of a conditioned response. In Neural Mechanism of Goal-Directed Behavior and Learning, R. F. Thompson, L. H. Hicks, and V. B. Shvyrkov, eds., Academic, New York.

Cohen, D. H. (1982) Central processing time for a conditioned response in a vertebrate model system. In Conditioning, C. D. Woody, ed., Plenum, New York.

Cohen, D. H. (1984) Identification of vertebrate neurons modified during learning: Analysis of sensory pathways. In Primary Neural Substrates of Learning and Behavioral Change, D. L. Alkon and J. Farley, eds., Cambridge, Cambridge, UK.

Cohen, D. H., and D. M. Goff (1978) Conditioned heart rate change in the pigeon: Analysis and prediction of acquisition patterns. Physiol. Psychol. 6: 127-141.

Cohen, D. H., and R. L. Macdonald (1971) Some variables affecting orienting and conditioned heart-rate responses in the pigeon. J. Comp. Physiol. Psychol. 74: 123-133.

Cohen, D. H., C. M. Gibbs, J. Siegelman, P. Gamlin, and J. Broyles (1982) Is locus coeruleus involved in plasticity of lateral geniculate neurons during learning? Neurosci. Abstr. 8: 666 .

Disterhoft, J. F., and D. K. Stuart (1977) Differentiated short latency response increases after conditioning in inferior colliculus neurons of alert rat. Brain Res. 130: 315-333.

Dubin, M. W., and B. G. Cleland (1977) Organization of visual inputs to interneurons of lateral geniculate nucleus of the cat. J. Neurophysiol. $40: 410-427$

Foote, S. L., F. E. Bloom, and G. Aston-Jones (1983) Nucleus locus coeruleus: New evidence of anatomical and physiological specificity. Physiol. Rev. 63: 844-914.

Gabriel, M., J. D. Miller, and S. E. Saltwick (1976) Multiple unit activity of the rabbit medial geniculate nucleus in conditioning, extinction, and reversal. Physiol. Psychol. 4: 124-134.

Gibbs, C. M., and D. H. Cohen (1980) Plasticity of the thalamofugal pathway during visual conditioning. Neurosci. Abstr. 6:424

Gibbs, C. M., D. H. Cohen, J. Broyles, and A. Solina (1981) Conditioned modification of avian dorsal lateral geniculate neurons is a function of their response to the unconditioned stimulus. Neurosci. Abstr. 7: 752 .

Gibbs, C. M., J. L. Broyles, and D. H. Cohen (1983) Further studies of the involvement of locus coenuleus in plasticity of avian lateral geniculate neurons during learning. Neurosci. Abstr. 9: 641 .

Gold, M. R., and D. H. Cohen (1981a) Modification of the discharge of vagal cardiac neurons during conditioned heart rate change. Science 214: 345-347.

Gold, M. R., and D. H. Cohen (1981b) Heart rate conditioning in the pigeon immobilized with $\alpha$-bungarotoxin. Brain Res. 216: 163-172.

Gold, M. R., and D. H. Cohen (1984) Discharge characteristics of vagal cardiac neurons during classically conditioned heart rate change. J. Neurosci. 4: 2963-2971.

Guillery, R. W. (1971) Patterns of synaptic interconnections in the dorsal lateral geniculate nucleus of cat and monkey. Vision Res. (Suppl.) 3: 211-227.

Hoffer, B. J., G. R. Siggins, A. P. Oliver, and F. E. Bloom (1973) Activation of the pathway from locus coeruleus to rat cerebellar Purkinje neurons: pharmacological evidence of noradrenergic central inhibition. J. Pharmacol. Exp. Ther. 184: 553-569.

Kandel, E. R., and J. H. Schwartz (1982) Molecular biology of learning: Modulation of transmitter release. Science 218: 433-443.

Karten, H. J. (1979) Visual lemniscal pathways in birds. In Neural Mechanisms of Behavior in the Pigeon, A. M. Granda and J. $\mathrm{H}$. Maxwell, eds., Plenum, New York. 
Karten, H. J., W. Hodos, W. J. H. Nauta, and A. M. Revzin (1973) Neural connections of the "visual wulst" of the avian telencephalon. Experimental studies in the pigeon (Columba livia) and owl (Speotyto cunicularia). J. Comp. Neurol. 150: 253-277.

Kettner, R. E., and R. F. Thompson (1982) Auditory signal detection and decision processes in the nervous system. J. Comp. Physiol. Psychol. 96: 328-331.

Killackey, H. P., and G. R. Belford (1980) Central correlates of peripheral pattern alterations in the trigeminal system of the rat. Brain Res. 183: 205-210.

Merzenich, M. M., R. J. Nelson, M. P. Stryker, M. S. Cynader, A Schoppmann, and J. M. Zook (1984) Somatosensory cortical map changes following digit amputation in adult monkeys. J. Comp. Neurol. 224: 591-605.

Oleson, T. D., J. H. Ashe, and N. M. Weinberger (1975) Modification of auditory and somatosensory system activity during pupillary conditioning in the paralyzed cat. J. Neurophysiol. 38: 1114-1139.

Ryugo, D. K., and N. M. Weinberger (1978) Differential plasticity of morphologically distinct neuron populations in the medial geniculate body of the cat during classical conditioning. Behav. Biol. 22: 275301 .
Sherman, S. M., and P. D. Spear (1982) Organization of visual pathways in normal and visually deprived cats. Physiol. Rev. 62: 738855.

Thompson, R. F., T. W. Berger, and J. Madden (1983) Cellular processes of learning and memory in the mammalian CNS. Annu. Rev. Neurosci. 6: 447-491.

Thompson, R. F., J. D. Barchas, G. A. Clark, N. Donegan, R. E. Kettner, D. G. Lavond, J. Madden IV, M. D. Mauk, and D. A. McCormick (1984) Neuronal substrates of associative learning in the mammalian brain. In Primary Neural Substrates of Learning and Behavioral Change, D. L. Alkon and J. Farley, eds., Cambridge, Cambridge, UK.

Tsukahara, N. (1982) Classical conditioning mediated by the red nucleus in the cat. In Conditioning, C. D. Woody, ed., Plenum, New York.

Wall, J. T., C. M. Gibbs, J. L. Broyles, and D. H. Cohen (1985) Modification of neuronal discharge along the tectofugal pathway during visual conditioning. Brain Res. 342: 67-76.

Wild, J. M., and D. H. Cohen (1985) Invariance of retinal output during visual learning. Brain Res. 331: 127-135. 